\title{
Criatividade em indivíduos com transtornos e dificuldades de aprendizagem: revisão de pesquisas
}

\author{
Rauni Jandé Roama Alves \\ Pontifícia Universidade Católica de Campinas - SP \\ Tatiana de Cássia Nakano \\ Pontifícia Universidade Católica de Campinas - SP
}

\begin{abstract}
Resumo
O presente trabalho teve como objetivo identificar as produções científicas nacionais e internacionais que tiveram como foco a investigação da relação entre criatividade e dificuldades/transtornos de aprendizagem. Em nível nacional foram consultadas as bases de dados SCIELO, PEPSIC, CAPES e BDTD e no internacional, as bases APA, ERIC, PubMed e Scopus, sem delimitação de tempo. Como resultado, constatou-se a existência de 57 produções internacionais e cinco nacionais. As análises identificaram que não houve diferenças estatísticas significativas entre as médias das produções das últimas três décadas e que as investigações focalizaram principalmente o transtorno específico de leitura/Dislexia, o uso da metodologia quantitativa e o emprego do Teste de Torrance. Alguns nomes dados aos grupos investigados na maioria dos trabalhos não denotaram com clareza as origens das dificuldades de aprendizagem - se seriam ou não decorrentes de transtorno de aprendizagem. Concluiuse que são necessários novos estudos sobre a temática, nos quais se caracterizem melhor os grupos investigados e a nomenclatura utilizada.
\end{abstract}

Palavras-chave: Pesquisa científica; distúrbios da aprendizagem; distúrbios do desenvolvimento.

\section{Creativity in individuals with disorders and learning disabilities: research review}

\begin{abstract}
This study aimed to characterize the national and international scientific production focused on investigating the relationship between creativity and difficulties / learning disabilities. Nationally were consulted the SCIELO databases, PEPSIC, CAPES and BDTD and internationally the APA bases, ERIC, PubMed and Scopus without delimitation of time. The results indicated the existence of 57 international and five domestic productions. The analysis found that there were no significant statistical differences between the averages of the past three decades productions; the investigations have focused mainly on the specific reading disability/Dyslexia; use of quantitative methodology; and using the Torrance Test. Some classifications given to groups investigated in most studies do not denote clearly the origins of learning disabilities, whether they were or not resulting from the learning disorder. We conclude that further studies are needed on the subject, provided there is a better characterization of the investigated groups and classifications used.
\end{abstract}

Keywords: Scientific research; learning disorders; developmental disorders.

\section{Creatividad en individuos con trastornos y dificultades de aprendizaje: revisión de investigación}

\begin{abstract}
Resumen
El presente estudio tuvo como objetivo caracterizar la producción científica nacional e internacional centrada en la investigación de la relación entre creatividad y dificultades/trastornos de aprendizaje. Nacionalmente se consultaron las bases de datos SCIELO, PEPSIC, CAPES y BDTD e internacionalmente de las bases APA, ERIC, PubMed y Scopus, sin delimitación de tiempo. Los resultados indicaron la existencia de 57 producciones internacionales y cinco nacionales. Los análisis identificaron que no hubo diferencias estadísticas significativas entre las medias de las producciones de las últimas tres décadas; investigaciones más centrada en el trastorno específico de la lectura/Dislexia; uso de la metodología cuantitativa; y la utilización del Test de Torrance. Algunas nomenclaturas dadas a los grupos investigados en la mayoría de los trabajos no denotaron con clareza as orígenes de las dificultades de aprendizaje, si serían o no derivados de trastorno de aprendizaje. Se concluye que son necesarios nuevos estudios sobre la temática, desde que haya mejor caracterización de los grupos investigados y de las nomenclaturas utilizadas.
\end{abstract}

Palabras-clave: investigación científica; disturbios del aprendizaje; disturbios del desarrollo. 


\section{Introdução}

De acordo com a definição estabelecida pelo National Joint Comittee of Learning Disabilities, "transtornos de aprendizagem" é um termo global que diz respeito a um grupo de dificuldades referentes à aquisição e uso de habilidades acadêmicas como leitura, escrita e matemática. Tais alterações seriam intrínsecas ao ser humano e assim estariam presentes ao longo de toda a vida do indivíduo que as possui (Muñoz, Fresneda, Mendonza, Carballo \& Pestun, 2005). Além disso, apresentam caráter crônico e podem acarretar comorbidades emocionais como depressão e transtornos de ansiedade, necessitando de intervenções específicas e adequadas a cada caso (Germano, \& Capellini, 2011; Lima, Salgado \& Ciasca, 2011).

Os transtornos de aprendizagem são decorrentes de disfunções do sistema nervoso central e relacionados a uma "falha" no processo de aquisição e processamento da informação, diferindo das "dificuldades de aprendizagem", pois este último quadro decorre de questões relacionadas a problemas de ordem pedagógica, emocional ou sociocultural ou a quadros neurológicos (Ciasca, 2003; Rubinstein, 1999).

No Manual de Diagnóstico e Estatístico das Perturbações Mentais (DSM-IV-TR) (American Psychiatric Association, APA, 2002a) são descritos quatro tipos de transtornos de aprendizagem: transtorno da leitura, transtorno da matemáti$\mathrm{ca}$, transtorno da expressão escrita e transtorno da aprendizagem sem outra especificação. Diferentemente, a Classificação Internacional de Doenças e Problemas Relacionados à Saúde (CID-10) (Organização Mundial da Saúde, OMS, 2008) utiliza outra denominação: "Transtornos específicos do desenvolvimento das habilidades escolares". Nesse manual são descritos seis tipos de transtornos: transtorno específico da leitura, transtorno específico da soletração, transtorno específico da habilidade aritmética, transtorno misto de habilidades escolares, outros transtornos do desenvolvimento das habilidades escolares e transtorno não especificado do desenvolvimento das habilidades escolares. Comumente na literatura e nas áreas aplicadas também se encontram a denominações "distúrbios de aprendizagem", "dislexia" (para transtorno de leitura), "discalculia" (para transtorno de matemática) e "disgrafia" (para transtorno de escrita) (APA, 2002a).

Os principais diagnósticos para esses transtornos, presentes em ambos os manuais (APA, 2002a; OMS, 2008), são: dificuldades de aprendizagem desde as primeiras etapas de desenvolvimento; desempenho abaixo do esperado para a idade em testes padronizados de leitura, matemática ou expressão escrita; não se devem a fatores como falta de oportunidade de aprendizagem, escolarização, deficiência intelectual ou déficits sensoriais.

Ainda envolvendo a temática dos transtornos de aprendizagem, mas dentro de uma visão que pode ser abarcada como característica da Psicologia Positiva, investigações sobre uma possível "dupla excepcionalidade" nesses casos também têm sido realizadas (Schultz, 2009). De acordo com Nakano e Siqueira (2012), a definição do conceito de "dupla excepcionalidade" envolve a ideia de que habilidades cognitivas podem apresentar desenvolvimentos muitas vezes considerados opostos, ou seja, pessoas que demonstram capacidades superiores em uma ou mais áreas podem, ao mesmo tempo, apresentar também deficiências ou condições incompatíveis com essas características.

Para Guimarães e Alencar (2013), o avanço dos estudos sobre essa dupla condição tem chamado a atenção de pesquisadores e profissionais interessados no desenvolvimento do indivíduo, de modo que, ainda segundo as autoras, “(...) torna-se necessário compreender a dupla excepcionalidade a partir de uma visão multidimensional, levando em consideração o desenvolvimento global do indivíduo" (p.109). Como exemplo dessas pesquisas que vêm sendo conduzidas, autores como Neihart (2008) e Ourofino (2007) citam casos de crianças que, mesmo sendo superdotadas/talentosas, apresentam, concomitantemente, sérios problemas emocionais, ou mesmo transtornos do desenvolvimento, como síndrome de Asperger, transtorno de déficit de atenção e hiperatividade, deficiências físicas e transtornos de aprendizagem.

Em face da complexidade do quadro e do recente interesse por ele, tem-se notado que a identificação de indivíduos duplamente excepcionais vem se mostrando uma tarefa difícil, pelas seguintes razões, apontadas por Sousa (2009): foco exagerado na avaliação da dificuldade, expectativas estereotipadas de desempenho, déficits em oportunidades e experiências, visões restritas de superdotação e preocupações específicas com a deficiência. Com estas características tais indivíduos se tornam mais facilmente alvos de rotulação negativa do que candidatos à superdotação.

Como a criatividade é tida como um dos elementos constituintes das altas habilidades dentro dos modelos explicativos mais atuais do fenômeno, como entendem Feldman (2000), Jarosewich, Pfeiffer e Morris (2002), Li e cols. (2009), Robinson e Clinkenbeard (2008), a presente pesquisa teve como objetivo identificar a produção científica, nacional e internacional, cujo foco tenha sido a relação entre criatividade e transtornos e dificuldades de aprendizagem. A relevância desse tipo de estudo deve-se à constatação de que a análise das produções científicas sobre uma determinada área permite a clarificação dos assuntos que têm recebido maior destaque entre os pesquisadores, do tipo de investigação que vem sendo privilegiado, dos instrumentos mais utilizados e da qualidade e efetividade dessas investigações (Méis, \& Letas, 1996; Witter, 1999). Do mesmo modo possibilitam a identificação de lacunas que necessitam de maiores investigações, pois fornecem um mapeamento das contribuições, necessidades e déficits da produção (Ferreira, 2002; Lustoza, Oliveira, \& Mello, 2010).

\section{Método}

\section{Material}

Oito bases eletrônicas de dados foram consultadas, das quais quatro são internacionais e quatro, nacionais. 
As internacionais são American Psychological Association (APA), Education Resources Information Center (ERIC), PubMed e Scopus, e as nacionais são Scientific Electronic Library Online (SCIELO), Periódicos Eletrônicos de Psicologia (PEPSIC), base de teses e dissertações da Coordenação de Aperfeiçoamento de Pessoal de Nível Superior (CAPES) e Biblioteca Digital de Teses e Dissertações (BDTD). Por meio destas bases foram levantadas e categorizadas todas as suas produções encontradas sobre a temática, em um total de 57 produções internacionais e cinco nacionais.

\section{Procedimento}

Nas base nacionais o termo "criatividade" foi cruzado com os termos propostos pelos manuais DSM-IV-TR (APA, 2002a) e CID-10 (OMS, 2008): distúrbio de aprendizagem, distúrbio de leitura, transtorno de matemática, transtorno de expressão escrita, distúrbio de aprendizagem não especificado, distúrbios específicos de desenvolvimento de habilidades escolares, distúrbio específico de leitura, transtorno específico da soletração, transtorno específico de habilidades aritméticas, transtorno misto de habilidades escolares, outros transtornos de desenvolvimento da escolástica, habilidades e transtorno do desenvolvimento das habilidades escolares não especificado. Do mesmo modo também foi utilizada a combinação de criatividade com os termos "dificuldades de aprendizagem", "transtornos de aprendizagem", "distúrbios de aprendizagem", "dislexia", "discalculia" e "disgrafia". O mesmo procedimento foi adotado na consulta às bases internacionais, havendo somente a tradução dos termos para a língua inglesa. Não foi estipulada limitação de tempo para todas essas buscas, porém vale ressaltar que a presente pesquisa foi realizada em março de 2012.

A princípio todas as produções encontradas foram lidas com o objetivo de selecionar somente aquelas que enfocassem a investigação de relações entre, de um lado, transtornos e dificuldades de aprendizagem, e do outro, a criatividade, descartando-se todas as demais. Na base da APA foram encontrados 192 artigos e selecionados 41; na da ERIC foram encontrados 131 artigos e selecionados 19; na PubMed foram encontrados 86 artigos e 10 foram selecionados; na Scopus foram encontrados 54 artigos e 14 foram selecionados. Durante a seleção dos artigos observou-se a existência de trabalhos indexados em duas ou mais bases de dados, mas esses trabalhos foram considerados uma única vez. Dessa forma, somadas as produções das quatro bases internacionais, obtiveram-se 84 artigos, mas com a retirada dos que se repetiam, restaram 57.

Em relação às bases nacionais, na CAPES foram encontradas 115 produções e selecionadas duas dissertações e uma tese; na BDTD foram encontradas 29 produções e apenas uma tese foi selecionada; nas bases PEPSIC e SCIELO foi pesquisada somente a palavra "criatividade", em razão do baixo número de artigos encontrados com o cruzamento dos caracteres das outras bases de dados. Desse modo, na PEPSIC foram encontrados 146 artigos e apenas um foi selecionado; na SCIELO foram encontrados 523 artigos e três foram selecionados, porém, dois deles foram desconsiderados, pois eram resultantes da tese selecionada no banco da CAPES e assim uma só pesquisa. A partir dessas duas bases foram levantadas, então, mais duas produções, uma brasileira e uma colombiana, sendo esta última analisada entre as produções internacionais. Ao todo, restaram cinco produções brasileiras.

Depois de selecionadas, as pesquisas foram categorizadas de acordo com o ano de publicação, grupos investigados e/ou nomes dados aos grupos investigados que foram objeto da pesquisa, tipos de instrumentos utilizados, metodologia e faixas etárias. Em seguida essas categorias foram comparadas e analisadas segundo suas frequências e percentagens. A única dificuldade encontrada foi que na maioria das produções, principalmente nas internacionais, só foi possível ter acesso aos resumos das produções, fato que gerou dificuldades no levantamento de alguns dados, tais como os de faixas etárias, objetivos de pesquisa e instrumentos utilizados.

\section{Resultados e discussão}

O primeiro aspecto que pôde ser observado diz respeito à quantidade de produções na área: dos trabalhos encontrados que tratavam da temática em foco, apenas cinco eram de autores brasileiros, enquanto os internacionais somaram cinquenta e sete.

Em relação aos anos de publicação primeiramente foram encontradas produções de 1977 a 2011 (figura 1). Na década de 80 foi verificada a maior percentagem de produção, com 37\% $(n=22)$ do total de trabalhos analisados, seguida pela primeira década de 2000 , com $32,2 \%(n=20)$, e pela década de 90, com 24,1\% ( $n=15)$. De 1977 a 1979 foram encontrados quatro trabalhos, e em 2011 apenas um. A maior frequência de produção na década de 80 pode provavelmente ser explicada, de acordo com Morais (2001), pelo fato de nessa década estudos científicos sobre a criatividade terem ressurgido mais fortemente, após um período crítico de enfraquecimento de pesquisas na área ocorrido no final da década de 70. De toda forma, não foi verificada diferença estatística significativa entre as médias das produções das três décadas (teste Kruskal-Wallis; $x^{2}=1,56, p=0,45$ ).

Podem-se observar três picos de produção, um em cada década. O primeiro é observado no ano de 1980, com quatro publicações (6,4\%); o segundo em 1995, também com quatro publicações $(6,4 \%)$, e o terceiro em 2009 , com seis publicações $(9,6 \%)$. Apesar de nos últimos dois anos (2010 e 2011) serem encontradas poucas produções (uma em cada ano), o pico de produção de todo período investigado encontra-se no final da primeira década de 2000, o que vai ao encontro dos dados encontrados por Nakano e Wechsler (2007). Isto confirma a percepção de Starko (2010) de que nos últimos anos houve um aumento considerável de estudos sobre criatividade no âmbito educacional (Silva, \& Nakano, 2012; Nakano, \& Wechsler, 2012; Nakano, 2011). 


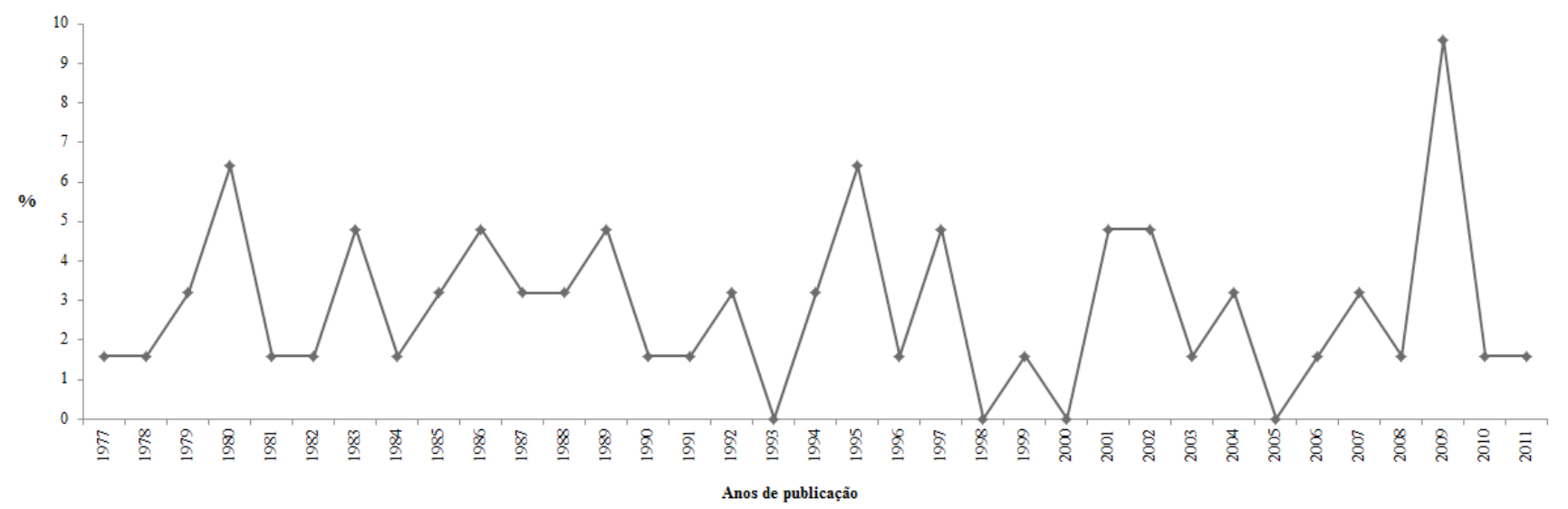

Figura 1. Percentagem das produções por ano

Outro importante dado observado refere-se aos nomes dados aos grupos pesquisados, tanto nas produções internacionais quanto nas nacionais. Em relação aos grupos brasileiros observou-se, nos cinco trabalhos, a denominação "dificuldades de aprendizagem". Nas produções internacionais os grupos pesquisados foram tidos, em sua maioria como afetados por "Learning Disabled"/", Learning Disabilities" e "Learning Disorders" (64,8\%), termos que traduzidos para o português se referem a transtornos de aprendizagem, de acordo com o que é encontrado nos manuais diagnósticos internacionais (APA, 2002b; World Health Organization, WHO, 2001). Foram também encontradas as denominações "Learning Difficulties" (dificuldades de aprendizagem) em $3,5 \%$ da amostra, "Retraso escolar" (atraso escolar) em $1,7 \%$ e "Dyslexia" (dislexia) em 29,8\%.

Quanto a estas denominações tem-se notado um problema que a área dos transtornos de aprendizagem vem enfrentando: comumente os termos "dificuldade de aprendizagem" e "transtornos de aprendizagem" têm sido utilizados como sinônimos na literatura nacional e internacional (Carvalho, Crenitte, \& Ciasca, 2007; Moojen, 1999; Rubinstein, 1999), o que causa confusões no meio científico. Dessa forma, para eliminar possíveis confusões, em todos os trabalhos aqui analisados foi dada especial atenção à leitura da parte metodológica, a fim de que fossem observadas referências à etiologia das dificuldades dos sujeitos investigados.

Mesmo em se tratando de trabalhos internacionais que utilizaram as denominações propostas pelos manuais internacionais, na grande maioria dos casos não foram referidos processos de avaliação e etiologias relativas a transtornos de aprendizagem. As denominações internacionais utilizadas permitem inferir que os trabalhos internacionais encontrados foram realizados, em sua maioria, com indivíduos portadores de transtornos de aprendizagem. Em nenhum dos estudos que utilizaram os termos "learning difficulties", "retraso escolar" e "dificuldade de aprendizagem" foram encontradas referências à etiologia das dificuldades, com os sujeitos selecionados somente por meio de um teste padronizado de desempenho escolar e/ou relato dos pro- fessores. Pôde-se observar, ainda, que o único transtorno específico de aprendizagem investigado - e o foi somente nas publicações internacionais - foi a dislexia. Não foram encontradas pesquisas sobre os outros transtornos específicos (discalculia - dyscalculia; disgrafia - dysgraphia).

Estes resultados deixam clara a necessidade de um olhar crítico sobre as produções sobre dificuldades e transtornos de aprendizagem, de modo que sejam melhor caracterizados os sujeitos investigados, para que os resultados obtidos possam ser melhor generalizáveis para cada grupo em específico.

Categorizaram-se também os objetivos das pesquisas, e em apenas um trabalho internacional não foi possível identificar esse tópico. Puderam ser observadas seis principais categorias de objetivos: (1) Investigar o desempenho criativo por meio de instrumentos psicológicos; (2) Verificar efeitos de programas de criatividade; (3) Discutir teoricamente relações entre criatividade e dificuldades/transtornos de aprendizagem; (4) Analisar estudos de caso; (5) Avaliar a criatividade por meio de juízes especialistas na área; (6) Fazer levantamento bibliográfico sobre a temática.

O objetivo mais visado nas investigações internacionais foi o de "Investigar o desempenho criativo por meio de instrumentos psicológicos", com 31,5\% ( $n=18)$. Ainda dentro dessa categoria puderam-se observar subgrupos de objetivos, tais como: comparar o desempenho criativo de indivíduos com dificuldades/transtornos de aprendizagem com outras habilidades cognitivas; verificar características criativas de indivíduos com dificuldades/transtornos de aprendizagem por meio de testes cognitivos mais abrangentes que possuíam também a avaliação dessa habilidade; comparar o desempenho criativo de crianças com dificuldades/transtornos de aprendizagem com outros grupos (por exemplo, comparar o desempenho de crianças superdotadas e que também possuíam dificuldades/transtornos de aprendizagem com o de crianças somente superdotadas).

Outro subgrupo de objetivos observado foi o de comparar o desempenho criativo de crianças que possuíssem dificuldades/transtornos de aprendizagem com o de crianças sem dificuldades de aprendizagem por meio de teste 
validado para essas populações pesquisadas. Tal subgrupo foi referente a oito produções do total das 18 internacionais que compuseram essa primeira categoria, sendo o mais frequente. Em dois desses oito trabalhos teve-se acesso somente aos resumos, e nestes não havia informações sobre seus procedimentos nem sobre os resultados. Em todos os outros seis foi utilizado o "Teste de Pensamento Criativo de Torrance", tanto verbal quanto figural, como instrumento de medida para a criatividade; porém os resultados encontrados não apresentam um padrão de achados: dois deles não encontraram diferenças entre os desempenhos criativos de ambos os grupos, outros dois verificaram que crianças com transtornos de aprendizagem apresentaram melhor desempenho em originalidade, um deles verificou que crianças com transtorno apresentaram melhor fluência e um encontrou que tais crianças foram piores em elaboração. $O$ único trabalho brasileiro pertencente à primeira categoria também se enquadrou nesse subgrupo, e nele também foi utilizado o teste Torrance, não sendo encontradas diferenças significativas entre os grupos de sujeitos que tinham dificuldades de aprendizagem e o grupo dos sem dificuldades.

Pesquisas sobre o efeito de programas de criatividade ocuparam a segunda posição entre as mais frequentes internacionalmente, com $26,3 \%(n=15)$, e a mais frequente nacionalmente, com $60 \%(n=3)$. Foi possível observar que na grande maioria das pesquisas internacionais $(n=13)$ a criatividade se mostrou como uma habilidade que pode ser desenvolvida; nas outras duas pesquisas encontradas não foi possível identificar os resultados em razão de terem sido localizados somente os resumos e estes não apresentarem tais dados. O pré e o pós-teste foram comumente avaliados por instrumentos validados $(n=13)$. Em nove deles foi utilizado o "Teste de Pensamento Criativo de Torrance", mas os achados também não apresentaram um padrão: em dois dos trabalhos não foi possível encontrar a sessão de resultados, somente eram comentados os ganhos de modo geral; em outros dois foram verificados ganhos em todas as habilidades criativas avaliadas pelo teste; em mais dois, melhoras em algumas habilidades criativas figurais (sendo comum entre ambas produções a originalidade); e em três foram observadas melhoras em algumas habilidades criativas verbais, sendo comuns entre as três a fluência e a flexibilidade. Nas produções nacionais também foi utilizado o teste de Torrance: em um dos trabalhos verificaram-se ganhos em habilidades criativas figurais (fluência e elaboração) e nos outros dois verificaram-se maiores ganhos em habilidades criativas verbais (principalmente em fluência). Dessa forma, apesar da inconstância dos resultados dos estudos - tanto internacionais quanto nacionais - verificaram-se ganhos em habilidades criativas após a aplicação de um programa para sua estimulação, o que concorda com a literatura, segundo a qual a criatividade pode ser desenvolvida (Alencar, \& Fleith, 2003; Dias, Enumo \& Azevedo, 2004; Feldman, 2000; Nakano, 2011; Novaes, 1972).

Sobre a questão da falta de consenso em relação aos resultados desses programas de estimulação, além de se verificar uma grande divergência entre os tipos de progra- mas existentes, também a metodologia adotada varia muito em termos de amostras e dos tipos de pesquisas realizados (Oliveira, 2007). Cropley (1999) destaca que os resultados do treinamento criativo que têm sido apontados na literatura científica não têm se mostrado simples e diretos e muitas vezes são bastante diferentes entre si. Nesse sentido, Fernández e López (1998) reafirmam que a influência e a diversidade da metodologia de pesquisa utilizada possivelmente são responsáveis por tais resultados, assim como o são as condições em que os dados sobre critérios são obtidos, cujo foco foi alterado em função do conteúdo, do modelo de criatividade escolhido e da experiência acumulada na avaliação destes programas.

Por sua vez, estudos que objetivaram "discutir teoricamente relações entre criatividade e dificuldades/transtornos de aprendizagem" compuseram 17,5\% $(n=10)$ das pesquisas internacionais e $20 \%(n=1)$ das nacionais. Esses estudos apresentavam discussões baseadas na literatura especializada e, de modo geral, propunham reflexões críticas sobre as questões como: propostas para desenvolvimento da criatividade no ambiente educacional em crianças com dificuldades/transtornos de aprendizagem; aspectos neurobiológicos que possam ser comuns aos casos de dificuldades/transtornos de aprendizagem e a criatividade; processos qualitativos e quantitativos para identificação e compreensão da criatividade em casos de dificuldades/transtornos de aprendizagem; possíveis influências do ambiente ou de outras habilidades cognitivas sobre a criatividade de indivíduos com dificuldades/transtornos de aprendizagem - entre outros. Sobre a ênfase nos estudos teóricos, Neihart (2000) aponta a importância desse tipo de produção científica a respeito da superdotação associada à síndrome de Asperger, e generaliza essa afirmação para outros transtornos - como, no presente caso, para os de aprendizagem. Por outro lado, a autora critica também o fato de muitas vezes esse tipo de pesquisa se sobrepor em excesso às empíricas, sendo um cuidado a ser relevado pelos pesquisadores da área.

Os "estudos de caso" estiveram presentes em 14\% $(n=8)$ da amostra internacional. Ventura (2007) afirma que o método do "estudo de caso" é comumente utilizado para investigar de maneira intensiva uma situação/caso particular em pesquisas de cunho exploratório, sendo muito útil na geração de hipóteses. Além disso, alega que esse método possui valor científico reconhecido na produção de conhecimento de áreas como as ciências sociais, psicologia, medicina, entre outras. No presente estudo essas pesquisas foram analisadas independentemente enquanto uma categoria de objetivo, pois se verificou que apresentavam características únicas, como a variabilidade na utilização de análises quantitativas e qualitativas sobre algumas condições (escolares, cotidianas, genéticas, etc.) e a formulação de inúmeras hipóteses sobre o desenvolvimento de determinadas habilidades, como criatividade, inteligência, comportamentos, sentimentos, etc. Pôde-se observar um pequeno padrão nessas produções: $30 \%$ delas se propuseram a estudar casos de alguns gênios da humanidade partindo do princípio de que sofriam de dificuldades/transtornos de aprendizagem e de 
Tabela 1. Testes utilizados nas publicações analisadas

\begin{tabular}{|c|c|c|c|c|}
\hline \multirow{2}{*}{ Instrumentos utilizados } & \multicolumn{2}{|c|}{ Internacional } & \multicolumn{2}{|c|}{ Nacional } \\
\hline & $f$ & $\%$ & $f$ & $\%$ \\
\hline \multicolumn{5}{|l|}{ Criatividade } \\
\hline Teste de Pensamento Criativo de Torrance & 19 & 63,3 & 3 & 27,2 \\
\hline Torrance Test of Thinking Creatively in Action and Movement & 1 & 3,3 & 0 & 0 \\
\hline \multicolumn{5}{|l|}{ Aspectos Comportamentais } \\
\hline Matching Familiar Figures Test & 1 & 3,3 & 0 & 0 \\
\hline Piers-Harris Children's Self-Concept Scale & 1 & 3,3 & 0 & 0 \\
\hline Scales for Rating the Behavioral Characteristics of Superior Students Revised & 1 & 3,3 & 0 & 0 \\
\hline Test of Interpersonal Problem Solving & 1 & 3,3 & 0 & 0 \\
\hline Scale for Rating the Behavioral Characteristics of Superior Students-Renzulli & 1 & 3,3 & 0 & 0 \\
\hline \multicolumn{5}{|l|}{ Habilidades Escolares } \\
\hline Arlin-Hills Survey Toward School Learning Processes & 1 & 3,3 & 0 & 0 \\
\hline Teste de Desempenho Escolar & 0 & 0 & 2 & 18,1 \\
\hline Test of Written Language (TOWL) & 1 & 3,3 & 0 & 0 \\
\hline \multicolumn{5}{|l|}{ Inteligência } \\
\hline Escala de Inteligência Wechsler (WISC) & 1 & 3,3 & 1 & 9,0 \\
\hline Matrizes Progressivas Coloridas de Raven & 0 & 0 & 2 & 18,1 \\
\hline \multicolumn{5}{|l|}{ Habilidades Cognitivas } \\
\hline Jogo de Perguntas de Busca de Figuras Diversas (PBFD) & 0 & 0 & 1 & 9,0 \\
\hline Cornoldi Test of Visual-Spatial and Verbal Memory & 1 & 3,3 & 0 & 0 \\
\hline Teste Gestáltico Visomotor de Bender & 0 & 0 & 1 & 9,0 \\
\hline Edinburgh Inventory & 1 & 3,3 & 0 & 0 \\
\hline \multicolumn{5}{|l|}{ Personalidade } \\
\hline Provas de Rorschach & 0 & 0 & 1 & 9,0 \\
\hline
\end{tabular}

que tal condição poderia ser hipoteticamente facilitadora ou até mesmo originadora dos talentos que possuíam.

Já pesquisas que tiveram como objetivo "avaliar a criatividade por meio de juízes especialistas na área" corresponderam a $7 \%$ do total da amostra internacional $(n=4)$. Esses tipos de pesquisa divergem do modelo psicométrico de avaliação da criatividade, já que a produção criativa é avaliada por juízes, comumente pesquisadores experientes na área. Em duas das pesquisas aqui analisadas os juízes verificaram que produções criativas figurais de sujeitos com dificuldades/transtornos de aprendizagem eram mais bem-elaboradas do que as dos grupos-controle, que não tinham essas dificuldades. As outras duas envolveram estudos com os próprios juízes - no caso, professores que trabalhassem na área -, para verificar se eram capazes de identificar habilidades criativas. Esse método de avaliação, intitulado Consensual Assessment Technique (CAT), consiste no julga- mento independente de produtos, por juízes especialistas, dentro de uma escala que varia entre o mais baixo e o mais alto nível de criatividade, partindo-se da percepção individual de cada juiz acerca do que é criatividade (Kaufman, Baer, Agars, \& Loomis, 2010). Esse tipo de avaliação é polêmico e diversas pesquisas internacionais têm sido conduzidas com o objetivo de verificar a importância/necessidade de juízes especialistas ou a possibilidade de resultados similares obtidos a partir do julgamento feito por não especialistas (Kaufman, Baer, Cole, \& Sexton, 2008; Kaufman, Lee, Baer, \& Lee, 2007). No Brasil, um estudo visando verificar a precisão de juízes no julgamento de produtos foi realizada por Primi, Miguel, Couto e Muniz (2007).

Por fim, o objetivo menos abordado foi o de "Levantamento bibliográfico sobre a temática", presente em $1,7 \%$ da amostra internacional $(n=1)$. Neste caso o estudo referiu-se especificamente ao levantamento de estudos que 
investigassem a relação entre dislexia e criatividade, sendo encontrado, de forma geral, que nesses casos: 1- as habilidades criativas apresentaram-se sensíveis à faixa etária nesses quadros, tendendo a aumentar conforme o desenvolvimento; 2- houve correlações diversas entre habilidades visoespaciais e criatividade figural; 3- foram poucas as investigações que puderam evidenciar o fator neurobiológico característico dos transtornos de aprendizagem como facilitador da apresentação de habilidades criativas (Everatt, Steffert, \& Smythe, 1999).

Outra análise realizada refere-se ao levantamento de instrumentos psicológicos utilizados nas pesquisas selecionadas, observado na Tabela 1. Os instrumentos mais frequentemente utilizados foram os que se prestavam para a avaliação da criatividade ( $f=23)$, seguidos por aqueles que avaliavam aspectos comportamentais, habilidades escolares, inteligência, habilidades cognitivas (todos com $f=4$ ). Por fim, o instrumento menos frequentemente utilizado referia-se à avaliação da personalidade $(f=1)$.

O "Teste de Pensamento Criativo de Torrance" foi o instrumento mais presente nas pesquisas internacionais $(57,5 \%)$ e nacionais $(27,2 \%)$. Outro instrumento que se fez presente tanto em pesquisas nacionais quanto em nacionais foi a "Escala de Inteligência Wechsler (WISC)". No Brasil ainda pode ser observada a utilização de um mesmo instrumento em diferentes pesquisas, no caso o instrumento "Matrizes Progressivas Coloridas de Raven" e o "Teste de Desempenho Escolar", o que não ocorre com as pesquisas internacionais.

A alta frequência de uso do "Teste Torrance de Pensamento Criativo" pode ser compreendida pelo fato de que, internacionalmente, é um teste consolidado de avaliação da criatividade, sendo sua validação existente em inúmeros países (Wechsler, 1998), o que corrobora os resultados aqui encontrados. No Brasil não é um teste validado para crianças, grupo ao qual o teste foi administrado, sendo permitido seu uso somente para pesquisas. Provavelmente isso deva à escassez de instrumentos validados para crianças na época em que foram realizadas as pesquisas. Atualmente existe um instrumento infantil, o Teste de Criatividade Figural Infantil (Nakano, Wechsler \& Primi, 2011), publicado recentemente.

Foram levantadas também as metodologias de pesquisa utilizadas nos trabalhos selecionados. Tanto em relação às publicações internacionais quanto às nacionais verificou-se que a metodologia quantitativa foi a mais utilizada, estando presente, respectivamente, em $57,8 \%(n=33)$ e em $80 \%(n=4)$ das amostras. Em segundo lugar encontram-se as publicações teóricas, com 19,2\% ( $n=11)$ nas publicações internacionais e $20 \%(n=1)$ nas nacionais que discutiam relações entre a criatividade e os dificuldades/transtornos de aprendizagem por meio de propostas de modelos explicativos (neuropsicológicos, desenvolvimentistas, etc.) e de proposições sobre o modelo de avaliação psicológica e também sobre a importância do reconhecimento de habilidades que possam ser mais bem desenvolvidas (no caso, a criatividade) em crianças que apresentam esse padrão de desempenho acadêmico. Somente em relação às pesquisas internacionais puderam ser observados outros tipos de metodologia, como a qualitativa $(15,7 \%, n=9)$ e a mista $(5,2 \%, n=3)$ (esta última envolve tanto a metodologia quantitativa quanto qualitativa). Houve uma pesquisa internacional (correspondente a $1,7 \%$ da amostra total) em que não pôde ser identificada a metodologia adotada. Esses resultados vão ao encontro ao que tem sido verificado em pesquisas atuais: que a abordagem quantitativa é uma das mais utilizadas para investigar a temática (Wechsler, \& Nakano, 2011; Zanella, \& Titon, 2005).

Verificaram-se também as faixas etárias dos trabaIhos analisados. Em relação aos trabalhos nacionais, tais dados puderam ser identificados em quatro dos cinco trabaIhos encontrados, já que se teve acesso apenas ao resumo de um deles e que este resumo não fornecia tal informação. Em todos os quatro foram investigados grupos de crianças. Em relação às publicações internacionais, verificou-se que grande parte delas também investigou e discutiu sobre grupos de crianças $(n=39)$, grupos de adolescentes $(n=9)$ e grupos de adultos $(n=8)$. Estes achados também corroboram o que tem sido encontrado, infelizmente, dentro do campo da pesquisa com criatividade, que é a baixa frequência de investigações com grupos minoritários (Nakano, \& Wechsler, 2007; Wechsler, 2001; Wechsler, \& Nakano, 2002). Especificamente em relação à presente temática, tais grupos seriam aqueles compostos por adultos e idosos.

\section{Considerações finais}

Com os procedimentos e materiais selecionados puderam ser atingidos os objetivos desta pesquisa. Primeiramente pôde-se verificar a necessidade de produções nacionais sobre a temática, já que nos mais de trinta anos investigados houve somente cinco produções. Outro dado foi que houve um aumento na frequência das pesquisas nos últimos anos, após um período de alta produtividade na década de 80 - apesar de, quer nacional quer internacionalmente, não ter-se podido verificar um padrão de achados relacionados à identificação de habilidades criativas em indivíduos com dificuldades/transtornos de aprendizagem, por exemplo, se apresentariam ou não melhores desempenhos criativos quando comparados a indivíduos sem dificuldades de aprendizagem. Por outro lado, verificou-se em inúmeros estudos que a criatividade pode ser desenvolvida, mesmo nesses casos.

Outra análise que se mostrou de extrema importância para as pesquisas sobre a temática diz respeito às denominações dadas aos grupos investigados. Todos os trabalhos brasileiros e alguns internacionais foram realizados com sujeitos tidos como portadores de "dificuldades de aprendizagem" - termo que, como se verificou, muitas vezes pode ser utilizado como sinônimo de transtornos de aprendizagem e assim causar confusões sobre qual quadro em especifico foi estudado, e até mesmo levar a conclusões errôneas sobre as áreas das dificuldades e transtornos de aprendizagem. Por isso se faz necessário padronizar os termos utilizados nas pesquisas, pois misto facilitara a compreensão dos indivíduos nelas envolvidos. 
De toda forma, uma limitação do presente trabalho é que somente foram consultadas bases de dados eletrônicas e que inúmeras produções podem não ter sido encontradas. Apesar disso, a amostra levantada forneceu possibilidades para uma análise do que vem sendo produzido e encontrado sobre a temática, assim como questões e procedimentos metodológicos que ainda devem ser respondidos e mais bem formulados para que haja conclusões mais sistematizadas sobre as relações entre as dificuldades e os transtornos de aprendizagem com a criatividade.

\section{Referências}

Alencar, E. M. L. S., \& Fleith, D. S. (2003). Criatividade: múltiplas perspectivas ( $3^{\mathrm{a}}$ ed.). Brasília: Universidade de Brasília.

American Psychiatric Association (APA). (2002a). DSM-IV-TR: Manual Diagnóstico e Estatístico de Transtornos Mentais (ed. rev.). Porto Alegre: Artes Médicas.

American Psychiatric Association (APA). (2002b). DSM-IV-TR, Diagnostic and statistical manual of mental disorders (ed. rev.). Washingon: APA.

Carvalho, F. B., Crenitte, P. A. P., \& Ciasca, S. M. (2007). Distúrbios de aprendizagem na visão do professor. Psicopedagogia, 24 (75), 229-239.

Ciasca, S. M. (2003). Distúrbios de aprendizagem: proposta de avaliação interdisciplinar. São Paulo: Casa do Psicólogo.

Cropley, A. J. (1999). Education. Em M. A. Runko, \& S. R. Pritzker (Orgs.), Encyclopedia of Creativity (Vol.1, pp. 629-642). San Diego, CA: Academic Press.

Dias, T. L., Enumo, S. R. F., \& Azevedo, R. R. (2004). Influências de um programa de criatividade no desempenho cognitivo e acadêmico de alunos com dificuldades de aprendizagem. Psicologia em Estudo, 9 (3), 429-437.

Everatt, J., Steffert, B., \& Smythe, I. (1999). An eye for the unusual: Creative thinking in dyslexics. Dyslexia, 5 (1), 28-46.

Feldman, D. H. (2000). The development of creativity. Em R. J. Sternberg (Org.), Handbook of creativity (pp. 169-189). Cambridge: Cambridge University Press.

Fernández, R. F., \& López, F. P. (1998). Estudio de tres modelos de creatividad: criterios para identificacion de la produccion creativa. Faisca: revista de altas capacidades, 6, 67-85.

Ferreira, N. S. A. (2002). As pesquisas denominadas estado da arte. Educação e Sociedade, 23 (79), 257-272.

Germano, G. D., \& Capellini, S. A. (2011). Desempenho de escolares com dislexia, transtornos e dificuldades de aprendizagem em provas de habilidades metafonológicas (PROHFON). Jornal da Sociedade Brasileira de Fonoaudiologia, 23 (2), 135-141.

Guimarães, T.G., \& Alencar, E.M.L.S. (2013). Estudo de caso de um aluno com características de superdotação e transtorno de Asperger. Em D.S. Fleith \& E.M.L.S. Alencar (Orgs.), Superdotados: trajetórias de desenvolvimento e realizações (pp. 109-120). Curitiba: Juruá Editora.

Jarosewich, T., Pfeiffer, S.I., \& Morris, J. (2002). Identifying gifted students using teacher rating scales: a review of existing instruments. Journal of Psychoeducational Assessment, 20, 322336.

Kaufman, J.C., Baer, J., Agars, M.D., \& Loomis, D. (2010). Creativity stereotypes and the consensual assessment techinique. Creativity Research Journal, 22 (2), 200-205.

Kaufman, J.C., Baer, J., Cole, J.C., \& Sexton, J.D. (2008). Comparison of expert and nonexpert raters using the consensual assessment technique. Creativity Research Journal, 20 (2), 171-178.

Kaufman, J.C., Lee, J., Baer, J., \& Lee, S. (2007). Captions, consistency, creativity and the consensual assessment technique: new evidence of reliability. Thinking Skills and Creativity, 2, 96-106.

Li, H., Lee, D., Pfeiffer, S. I., Kamata, A., Kumtepe, A. T., \& Rosado, J. (2009). Measurement invariance of the Gifted Rating Scales - school form across five cultural groups. School Psychology Quaterly, 4 (3), 186-198.

Lima, R. F. de, Salgado, C. A., \& Ciasca, S. M. (2011). Associação da dislexia do desenvolvimento com comorbidade emocional: um estudo de caso. Revista CEFAC, 13 (4), 756-762.

Lustoza, R. Z., Oliveira, K. L., \& Mello, B. N. (2010). Produção científica no contexto psicanalítico. Psico-USF, 15 (2), 161-169.

Méis, L., \& Leta, J. O perfil da ciência brasileira. Rio de Janeiro: UFRJ, 1996.

Moojen, S. (1999). Dificuldades ou transtornos de aprendizagem? Em E. Rubinstein (Org.), Psicopedagogia: uma prática, diferentes estilos (pp. 243-284). São Paulo: Casa do Psicólogo.

Morais, M. F. (2001). Definição e avaliação da criatividade. Braga: Universidade do Minho.

Muñoz, J., Fresneda, M. D., Mendonza, E., Carballo, G., \& Pestun, M. S. V. (2005). Descrição, avaliação e tratamento dos transtornos da aprendizagem. Em V. Caballo e M. Simón (Orgs.), Manual de psicologia clínica infantil e do adolescente (pp. 159-175). São Paulo: Editorial Santos.

Nakano, T. C. (2011). Programas de treinamento em criatividade: conhecendo as práticas e resultados. Psicologia Escolar e Educacional, 15 (2), 311-322. 
Nakano, T. C., \& Wechsler, S. M. (2007). Criatividade: características da produção científica brasileira. Avaliação Psicológica, 6 (2), 261270.

Nakano, T. C., \& Wechsler, S. M. (2012). Criatividade: definições, modelos e formas de avaliação. Em C. S. Hutz (Org.), Avanços em avaliação psicológica e neuropsicológica de crianças e adolescentes II (pp. 327-361). São Paulo: Casa do Psicólogo.

Nakano, T. C., Wechsler, S. M., \& Primi, R. (2011). Teste de criatividade figural infantil. São Paulo: Editora Vetor.

Nakano, T. D. C., \& Siqueira, L. G. G. (2012). Validade de conteúdo da Gifted Rating Scale (versão escolar) para a população brasileira. Avaliação Psicológica, 11 (1), 123-140.

Neihart, M. (2000). Gifted children with Asperger's syndrome. Gifted Child Quaterly, 44, 222-230.

Neihart, M. (2008). Identifying and providing services to twice exceptional children. Em S. Pfeiffer (Org.). Handbook of giftedness in children: Psycho-Educational theory, research and best practices (pp. 115-137). New York: Springer.

Novaes, M. H. (1972). Psicologia da criatividade. Petrópolis, RJ: Vozes.

Oliveira, E. P. (2007). Alunos sobredotados: a aceleração escolar como resposta educativa. Tese de Doutoramento, Universidade do Minho, Braga, Portugal.

Organização Mundial da Saúde (OMS). (2008). Classificação de transtornos mentais e de comportamento da CID-10: descrição clínicas e diretrizes diagnósticas (ed. rev.). Porto Alegre: Artes Médicas.

Ourofino, V. T. A. T. (2007). Altas habilidades e hiperatividade: a dupla excepcionalidade. Em D. S. Fleith, \& E. M. L. S. Alencar (Orgs.), Desenvolvimento de talentos e altas habilidades: orientação a pais e professores (pp. 51-66). Porto Alegre: Artmed.

Primi, R., Miguel, F.K, Couto, G., \& Muniz, M. (2007). Precisão de avaliadores na avaliação da criatividade por meio da produção de metáforas. PsicoUSF, 12 (2), 197-210.

Robinson, A., \& Clinkenbeard, P. R. (2008) History of giftedness: perspectives from the past presage modern scholarship. Em S. Pfeiffer (Org.), Handbook of giftedness in children: PsychoEducational theory, research and best practices (pp. 13-31). New York: Springer.
Rubinstein, E. (1999). Psicopedagogia: uma prática, diferentes estilos. São Paulo: Casa do Psicólogo.

Schultz, S. (2009). Twice-exceptional students participating in advanced placement. Saarbrucken, Germany: VDM.

Silva, T. F., \& Nakano, T. C. (2012). Criatividade no contexto educacional: análise de publicações periódicas e trabalhos de pós-graduação na área da psicologia. Educação e Pesquisa, 1-17.

Sousa, D. A. (2009). How the gifted brain learns. Califórnia: Thousand Oaks.

Starko, A. J. (2010). Creativity in the classroom - schools of curious delight. New York: Routledge.

Ventura, M. M. (2007). O estudo de caso como modalidade de pesquisa. Revista SoCERJ, 20 (5), 383-386.

Wechsler, S. M. (1998). Criatividade: descobrindo e encorajando. São Paulo: Editora Psy.

Wechsler, S. M. (2001). Criatividade na cultura brasileira: uma década de estudo. Revista portuguesa de psicologia: teoria, investigação e prática, 6 (1), 215-227.

Wechsler, S. M., \& Nakano, T. C. (2002). Caminhos para a avaliação da criatividade: perspectiva brasileira. Em R. Primi (Org.), Temas em avaliação psicológica (pp. 103-115). Campinas: IDB.

Wechsler, S. M., \& Nakano, T. C. (2011). Criatividade: encontrando soluções para os desafios educacionais. Em S. M. Wechsler, \& V. L. T. Souza (Orgs.), Criatividade e aprendizagem: caminhos e descobertas em perspectiva internacional (pp. 11-31). São Paulo: Edições Loyola.

Witter, G. P. (1999). Metaciência e leitura. Em G. P. Witter (Org.), Leitura: Textos e pesquisas (pp. 13 -22). Campinas: Alínea.

World Health Organization (WHO). (2001). ICD-10. International statistical classification of diseases and related health problems. Geneva: World Health Organization (WHO).

Zanella, A. V., \& Titon, A. P. (2005). Análise da produção científica sobre criatividade em programas brasileiros de pós-graduação em psicologia (1994-2001). Psicologia em Estudo, 10 (2), 305-316. 


\section{Sobre os autores}

Rauni Jandé Roama Alves (rauniroama@gmail.com)

Doutorando e Mestre em Psicologia pela PUC-Campinas.

Tatiana de Cássia Nakano (tatiananakano@hotmail.com)

Docente do programa de pós-graduação stricto sensu em Psicologia da PUC-Campinas.

Agradecimento: O primeiro autor agradece o apoio recebido do Conselho Nacional de Desenvolvimento Científico e Tecnológico (CNPq), sob a forma de bolsa de estudo. 